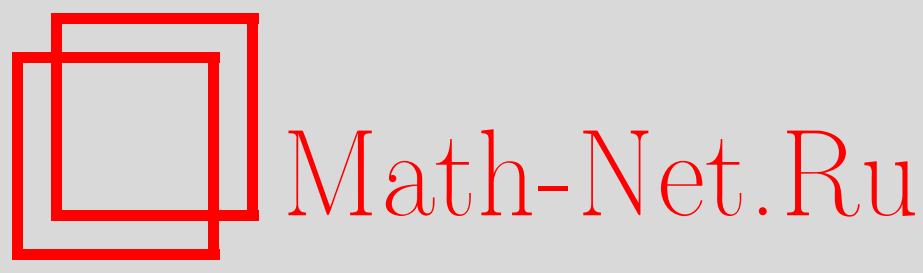

М. Палезе, Е. Уинтеррот, Вариационный взгляд на классические поля Хиггса в калибровочно-естественных теориях, ТМФ, 2011, том 168, номер 1, 171-179

DOI: https://doi.org/10.4213/tmf6672

Использование Общероссийского математического портала Math-Net.Ru подразумевает, что вы прочитали и согласны с пользовательским соглашением http: //www . mathnet.ru/rus/agreement

Параметры загрузки :

IP : 18.234 .156 .22

26 апреля 2023 г., 10:12:15

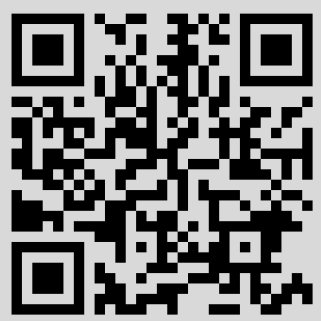




\section{ВАРИАЦИОННЫЙ ВЗГЛЯД НА КЛАССИЧЕСКИЕ ПОЛЯ ХИГГСА В КАЛИБРОВОЧНО-ЕСТЕСТВЕННЫХ ТЕОРИЯХ}

Поля Хиггса на естественно-калибровочных продолжениях главных расслоений определяются инвариантными вариационными задачами и соответствующими каноническими законами сохранения вдоль ядра калибровочно-естественного морфизма Якоби.

Ключевые слова: струи, калибровочно-естественное расслоение, сохраняющиеся величины, картанова связность, поле Хиггса.

\section{1. ВВЕДЕНИЕ}

В 1981 г. Ек [1] поместил естественные и калибровочные (классические) лагранжевы теории поля в рамки геометрического функториального построения, называемого калибровочно-естественным расслоением, в соответствии с которым физические поля считаются сечениями расслоений, функториально связанными с калибровочно-естественными продолжениями (также называемыми продолжениями Эресмана [2]) главных расслоений, с помощью левых действий групп Ли на многообразия, обычно тензорные пространства. Изучение струйных продолжений главных расслоений со структурной группой $\mathbf{G}$ (группой Ли) очень важно в физике. Однако подобное продолжение, как известно, не является главным расслоением, в то время как структура главного расслоения передается так называемому калибровочно-естественному продолжению главного расслоения [2], [3]. Говоря точнее, мы рассматриваем лагранжевы теории поля, которые считаются инвариантными по отношению к действию калибровочно-естественной группы $W_{n}^{(r, k)} \mathbf{G}$, определяемому как полупрямое произведение дифференциальной группы $k$-го порядка базисного многообразия на группу порядка $r n$-х скоростей в $\mathbf{G}$; здесь $n=\operatorname{dim} \mathbf{X}-$ размерность базисного многообразия. Так как группа $\operatorname{Diff}(\mathbf{X})$ не является канонически вложенной в группу $\operatorname{Aut}(\mathbf{P})$ всех автоморфизмов подлежащего главного расслоения $\mathbf{P}$, априори не существует естественного способа связать инфинитезимальные

*Department of Mathematics, University of Torino, Torino, Italy.

E-mail: marcella.palese@unito.it, ekkehart.winterroth@unito.it 
калибровочные преобразования с инфинитезимальными базисными преобразованиями, так что производные Ли калибровочного поля по отношению к инфинитезимальным базисным преобразованиям не могли бы определяться ни естественным, ни каноническим образом (по меньшей мере, априори).

Проблема существования ковариантных канонически определенных сохраняющихся токов связана с такими же свойствами производной Ли [4]. Хорошо известно, что ковариантность лагранжиана и уравнений Эйлера-Лагранжа не гарантирует соответствующую ковариантность нётеровских сохраняющихся величин. Вообще говоря, фиксирование линейной связности на базисном многообразии и главной связности на главном расслоении является необходимым для получения ковариантных сохраняющихся величин в калибровочно-естественных теориях поля (глобальная форма Пуанкаре-Картана может быть определена только с помощью фиксирования такой пары связностей; см., например, [5]). Однако мы обнаружили, что каноническое определение нётеровских сохраняющихся величин без фиксации какой-либо связности априори возможно не всегда на расслоении, получаемом из $W^{(r, k)} \mathbf{P}$ редукцией и задаваемом исходной $W_{n}^{(r, k)} \mathbf{G}$-инвариантной вариационной задачей. Связности могут характеризоваться с помощью такой канонической редукции [6]-[8]. Это происходит благодаря тому, что расслоения полей, связанные с классом главных расслоений, полученных как калибровочно-естественные продолжения главных расслоений, имеют более богатую структуру, чем расслоения, связанные с произвольными главными расслоениями.

Вариационная задача о струях расслоенных многообразий является инвариантной по отношению к контактной структуре конечного порядка, индуцированной струями: мы рассмотрим вариационные задачи с лагранжианом конечного порядка в терминах внешних дифференциалов форм по модулю контактной формы, сформулированной в контексте вариационных последовательностей конечного порядка [9]; более того, в категории вариационных последовательностей на калибровочно-естественных расслоениях производная Ли сечений расслоений (с точностью до знака) является вертикальной частью по отношению к контактной структуре (а не вертикальной компонентой по отношению к проекции на базисном многообразии) калибровочно-естественных подъемов инфинитезимальных главных автоморфизмов [10]. В предыдущей статье [11] в рамках вариационных последовательностей конечного порядка мы характеризовали вторую вариацию калибровочно-естественного инвариантного лагранжиана произвольного порядка и использовали эту характеристику для того, чтобы перенести на частный случай теоремы Нётер и соответствующие сохраняющиеся токи, а также обобщенные канонически определенные тождества Бергманна-Бианки для существования суперпотенциалов [12]. Мы решили проблему канонической ковариантности сохраняющихся величин с помощью вариационных производных, взятых по отношению к обобщенным векторным полям, которые выбирались как производные Ли сечений калибровочно-естественных расслоений, взятых по отношению к калибровочно-естественным подъемам инфинитезимальных главных автоморфизмов.

Законы сохранения, связанные с группой преобразований, полученных подстановкой параметров с произвольными функциями (бесконечные непрерывные группь) были изучены Нётер [13] в 1918 г., которая установила существование определенных тождественных соотношений между выражениями Эйлера-Лагранжа и их произ- 
водными как следствие инвариантности лагранжиана по отношению к такой более широкой группе преобразований (тождества Нётер). В 1956 г. Утияма [14] рассмотрел некоторые системы полей, инвариантных по отношению к определенной группе преобразований, зависящих от $n$ параметров, и, постулируя инвариантность таких систем по отношению к более широкой группе, полученной заменой параметров на набор произвольных функций, он ввел новое поле с определенным типом взаимодействия с исходными полями, определенными с помощью ковариантной производной.

С такой точки зрения мы рассматриваем класс параметризованных контактных преобразований, определяемых с помощью калибровочно-естественного функтора: обращаясь к инвариантным свойствам, мы определяем ковариантные производные полей и новые законы сохранения с помощью построения главной связности, удовлетворяющей определенному дополнительному условию. В частности, мы характеризуем канонические ковариантные сохраняющиеся величины лагранжиана в классической теории поля в терминах полей Хиггса на таких калибровочных главных расслоениях, обладающих более богатой структурой калибровочно-естественного продолжения. С этой точки зрения топологические условия существования картановой связности на главном расслоении $W^{(r, k)} \mathbf{P}$ оказываются необходимым условием существования глобальных решений уравнений Якоби, связанных с существованием канонически определенных глобальных сохраняющихся величин. В результате производная Ли полей является ограниченной и параметризуется с помощью поля Хиггса $h$, определенного пространством полей Якоби.

\section{2. ПОЛЯ ЯКОБИ, ПОРОЖДАЮЩИЕ КАНОНИЧЕСКИЕ ЗАКОНЫ СОХРАНЕНИЯ}

Напомним некоторые полезные понятия продолжений (подробнее см., например, [3], [15]). Пусть $J_{s} \mathbf{Y}-s$-струйное продолжение (локальных) сечений расслоенного многообразия $\pi: \mathbf{Y} \rightarrow \mathbf{X} \mathrm{c} \operatorname{dim} \mathbf{X}=n$ и $\operatorname{dim} \mathbf{Y}=n+m$. Естественные слоения $\pi_{s-1}^{s}$ являются афбинными слоениями, индуцирующими естественное расщепление (см., например, [9], [16])

$$
J_{s} \mathbf{Y} \times J_{s-1} \mathbf{Y} T^{*} J_{s-1} \mathbf{Y}=J_{s} \mathbf{Y} \times{ }_{J_{s-1}} \mathbf{Y}\left(T^{*} \mathbf{X} \oplus V^{*} J_{s-1} \mathbf{Y}\right),
$$

которое дает разложения возрастающего порядка: задано векторное поле $\Xi: J_{s} \mathbf{Y} \rightarrow$ $T J_{s} \mathbf{Y}, T \pi_{s}^{s+1} \circ \Xi=\Xi_{\mathrm{H}}+\Xi_{\mathrm{V}}$, где $\Xi_{\mathrm{H}}$ и $\Xi_{\mathrm{V}}-$ соответственно горизонтальная и вертикальная части поля $\Xi$; для внешнего дифференциала на $\mathbf{Y}$ мы имеем $\left(\pi_{r}^{r+1}\right)^{*} \circ d=$ $d_{\mathrm{H}}+d_{\mathrm{V}}$, где $d_{\mathrm{H}}$ и $d_{\mathrm{V}}$ называются горизонтальным и вертикальным дифференииалами соответственно; расщепление пучка записывается как $\mathcal{H}_{(s+1, s)}^{p}=\bigoplus_{t=0}^{p} \mathcal{C}_{(s+1, s)}^{p-t} \wedge$ $\mathcal{H}_{s+1}^{t}$, где $\mathcal{H}_{(s, q)}^{p}$ и $\mathcal{H}_{s}^{p}$ - пучки горизонтальных форм по отношению к проекциям $\pi_{q}^{s}$ и $\pi_{0}^{s}$ соответственно, в то время как $\mathcal{C}_{(s, q)}^{p} \subset \mathcal{H}_{(s, q)}^{p}$ и $\mathcal{C}_{s}^{p} \subset \mathcal{C}_{(s+1, s)}^{p}$ являются контактными формами, т. е. горизонтальными формами со значениями в $\mathcal{C}_{s}^{*}[\mathbf{Y}]$. Положим $\mathcal{H}_{s+1}^{p, h} \doteq h\left(\Lambda_{s}^{p}\right)$ при $0<p \leqslant n$, где проекция на слагаемое меньшей контактной степени $h$ является горизонтализацией.

Обозначим через $d$ ker $h$ пучок, порожденный предпучком $d$ ker $h$ стандартным образом. Положим $\Theta_{s}^{*} \doteq \operatorname{ker} h+d \operatorname{ker} h$. Мы имеем вариационную последовательность $0 \rightarrow \mathbb{R}_{Y} \rightarrow \mathcal{V}_{s}^{*}$, где $\mathcal{V}_{s}^{*}=\Lambda_{s}^{*} / \Theta_{s}^{*}$, что является точным расщеплением постоянного пучка $\mathbb{R}_{Y}[9]$. Сечение $E_{d \lambda} \doteq \mathcal{E}_{n}(\lambda) \in \mathcal{V}_{s}^{n+1}$ является обобщенным морфизмом стариего порядка типа Эйлера-Лагранжа, связанного с $\lambda$. 
Пусть $\mathbf{P} \rightarrow \mathbf{X}$ - главное расслоение со структурной группой $\mathbf{G}$. Для целочисленных $r \leqslant k$ рассмотрим калибровочно-естественное продолжение расслоения $\mathbf{P}$, задаваемое как $\mathbf{W}^{(r, k)} \mathbf{P} \doteq J_{r} \mathbf{P} \times \mathbf{X} L_{k}(\mathbf{X})$, где $L_{k}(\mathbf{X})$ - расслоение $k$-реперов в $\mathbf{X}$ [1], [3]; $\mathbf{W}^{(r, k)} \mathbf{P}$ - главное расслоение над $\mathbf{X}$ со структурной группой $\mathbf{W}_{n}^{(r, k)} \mathbf{G}$, которая является полупрямым произведением по отношению к действию $G L_{k}(n)$ на $\mathbf{G}_{n}^{r}$, задаваемому композицией струй, а $G L_{k}(n)$ - группа $k$-реперов в $\mathbb{R}^{n}$. Обозначим через $\mathbf{G}_{n}^{r}$ пространство $(r, n)$-скоростей на $\mathbf{G}$. Пусть $\mathbf{F}$ - многообразие, а $\zeta: \mathbf{W}_{n}^{(r, k)} \mathbf{G} \times \mathbf{F} \rightarrow \mathbf{F}$ - левое действие $\mathbf{W}_{n}^{(r, k)} \mathbf{G}$ на $\mathbf{F}$. Имеется естественное правое действие $\mathbf{W}_{n}^{(r, k)} \mathbf{G}$ на $\mathbf{W}^{(r, k)} \mathbf{P} \times \mathbf{F}$, так что мы имеем стандартным образом ассоциированное калибровочно-естественное расслоение порядка $(r, k): \mathbf{Y}_{\zeta} \doteq \mathbf{W}^{(r, k)} \mathbf{P} \times{ }_{\zeta} \mathbf{F}$. Во всех наших рассуждениях $\mathbf{Y}$ будет калибровочно-естественным расслоением, определенным выше.

Теперь обозначим через $\mathcal{A}^{(r, k)}$ пучок правоинвариантных векторных полей на $\mathbf{W}^{(r, k)} \mathbf{P}$. Калибровочно-естественный подбем определяется как функториальное отображение $\mathfrak{G}: \mathbf{Y}_{\zeta} \times \mathbf{x} \mathcal{A}^{(r, k)} \rightarrow T \mathbf{Y}_{\zeta}:(\mathbf{y}, \bar{\Xi}) \mapsto \widehat{\Xi}(\mathbf{y})$, где для любого $\mathbf{y} \in \mathbf{Y}_{\zeta}$ мы полагаем $\widehat{\Xi}(\mathbf{y})=\frac{d}{d t}\left[\left(\Phi_{\zeta t}\right)(\mathbf{y})\right]_{t=0}$, а $\Phi_{\zeta t}$ обозначает (локальный) поток, соответствующий калибровочно-естественному подъему автоморфизма $\Phi_{t}$. Такой функтор определяет класс параметризованных контактных преобразований. Это отображение удовлетворяет следующим свойствам (см. [3]): $\mathfrak{G}$ линейно над $i d_{\mathbf{Y}_{\zeta}}$; мы имеем $T \pi_{\zeta} \circ \mathfrak{G}=i d_{T \mathbf{X}} \circ \bar{\pi}^{(r, k)}$, где $\bar{\pi}^{(r, k)}-$ естественная проекция $\mathbf{Y}_{\zeta} \times \mathbf{X} \mathcal{A}^{(r, k)} \rightarrow T \mathbf{X}$; для любой пары $(\bar{\Lambda}, \bar{\Xi}) \in \mathcal{A}^{(r, k)}, \mathfrak{G}$ - гомоморфизм алгебр Ли.

Производная Ли является фундаментальным геометрическим объектом, дающим информацию о поведении решений уравнений Эйлера-Лагранжа при инфинитезимальных преобразованиях (автоморфизмах) калибровочно-естественного расслоения. Пусть $\gamma-$ (локальное) сечение расслоения $\mathbf{Y}_{\zeta}, \bar{\Xi} \in \mathcal{A}^{(r, k)}$, а $\widehat{\Xi}-$ его калибровочно-естественный подъем. Следуя работе [3], определим обобщенную производную Ли сечения $\gamma$ вдоль векторного поля $\widehat{\Xi}$ таким образом, что она является (локальным) сечением $£_{\bar{\Xi}} \gamma: \mathbf{X} \rightarrow V \mathbf{Y}_{\zeta}$, задаваемым выражением $£_{\bar{\Xi}} \gamma=T \gamma \circ \xi-$ $\widehat{\Xi} \circ \gamma$. Благодаря функториальной природе $\widehat{\Xi}$ производная Ли сечений наследует некоторые полезные свойства линейности, в частности она является гомоморфизмом алгебр Ли. Ввиду теорем Нётер, интерес к производным Ли сечений вызван тем, что в случае калибровочно-естественного подъема мы имеем $\widehat{\Xi}_{\mathrm{V}}=-£_{\Xi}$. Далее мы рассмотрим вариационные последовательности на калибровочно-естественном расслоении $\mathbf{Y}$.

Пусть $\eta \in \mathcal{C}_{s}^{1} \wedge \mathcal{C}_{(s, 0)}^{1} \wedge \mathcal{H}_{s+1}^{n, h}$, а $\Xi-$ вертикальное векторное поле; морфизм $\left.E_{\left.j_{s} \Xi\right\rfloor \eta}=\left(\pi_{s+1}^{2 s+1}\right)^{*} j_{s} \Xi\right\rfloor \eta+F_{\left.j_{s} \Xi\right\rfloor \eta}$ (где $F_{\left.j_{s} \Xi\right\rfloor \eta}-$ локальная дивергенция) является однозначно определенным глобальным сечением расслоения $\mathcal{C}_{(2 s, 0)}^{1} \wedge \mathcal{H}_{2 s+1}^{n, h}$ (см. [16]). Пусть $\lambda$ - лагранжиан, $\widehat{\Xi}_{\mathrm{V}}$ - обобщенное вариационное векторное поле, и $\eta=$ $h d \mathcal{L}_{j_{2 s} \bar{\Xi}_{\mathrm{V}}} \lambda$. Положим $\left.\chi\left(\lambda, \widehat{\Xi}_{\mathrm{V}}\right) \doteq E_{j_{s}} \widehat{\Xi}\right\rfloor \eta_{\eta}$. Применяя свойства функториальной линейности поля $\widehat{\Xi}$, определим линейный морфизм, калибровочно-естественный обобщенный морфизм Якоби, связанный с лагранжианом $\lambda$ и вариационным векторным полем $\widehat{\Xi}_{\mathrm{V}}, \mathcal{J}\left(\lambda, \widehat{\Xi}_{\mathrm{V}}\right) \doteq E_{\cdot \downarrow \chi}\left(\lambda, \widehat{\Xi}_{\mathrm{V}}\right)[10]$. Оказывается, что $\mathcal{J}\left(\lambda, \widehat{\Xi}_{\mathrm{V}}\right)$, вторая вариаци-

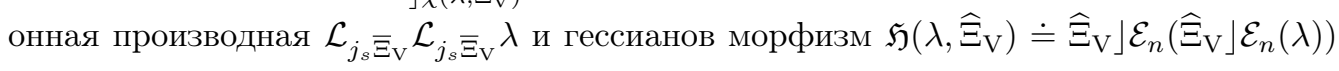
являются представителями одного и того же класса эквивалентности в соответствующей вариационной последовательности [11], таким образом характеризуя $\mathcal{J}\left(\lambda, \widehat{\Xi}_{\mathrm{V}}\right)$ 
как симметричный самосопряженный морфизм. Актуальность этого свойства связана с важными геометрическими аспектами пространства $\mathfrak{K} \doteq \operatorname{ker} \mathcal{J}\left(\lambda, \widehat{\Xi}_{\mathrm{V}}\right)$, определяющего обобщенные калибровочно-естественные уравнения Якоби, решения которых мы называем обобщенными векторными полями Якоби; они характеризуют канонические ковариантные сохраняющиеся величины [4].

Хорошо известно, что первую теорему Нётер можно записать в новой форме, если использовать вариационную производную Ли классов форм, представленных в вариационной последовательности: $\mathcal{L}_{j_{s} \Xi} \lambda=\vartheta\left(\lambda, £_{\Xi}\right)+d_{\mathrm{H}} \epsilon\left(\lambda, £_{\Xi}\right)$, где мы полагаем $\left.\vartheta\left(\lambda, £_{\Xi}\right) \doteq-£_{\Xi}\right\rfloor \mathcal{E}_{n}(\lambda)$, а $\epsilon\left(\lambda, £_{\Xi}\right)-$ нётеровский ток. Как обычно, $\lambda$ определяется как калибровочно-естественный инвариантный лагранжиан, если калибровочно-естественный подъем $(\widehat{\Xi}, \xi)$ любого векторного поля $\Xi \in \mathcal{A}^{(r, k)}$ является симметрией для $\lambda$ т. е. если $\mathcal{L}_{j_{s}} \bar{\Xi}=0$. Замечательно то, что в общем случае $\mathcal{L}_{j_{s}} \bar{\Xi}_{\mathrm{V}} \lambda \neq 0$.

Как уже отмечалось, существование канонического глобального суперпотенциала для $\epsilon\left(\lambda, £_{\Xi}\right)$ зависит от ковариантных тождеств Бергманна-Бианки, которые, как можно доказать, существуют канонически только вдоль $\operatorname{ker} \mathcal{J}\left(\lambda, \widehat{\Xi}_{\mathrm{V}}\right)$ [10]. Благодаря тому, что они являются тождествами Нётер, ассоциированными со свойствами инвариантности морфизма Эйлера-Лагранжа $\mathcal{E}_{n}(\vartheta)$, ядро $\mathfrak{K}$, будучи ядром гамильтонова оператора, может быть охарактеризовано как векторное подрасслоение [11], [17]. Для теории производной Ли калибровочно-естественных полей существенно то, что неопределенность сохраняющихся зарядов, свойственная им и связанная с калибровочно-естественными симметриями лагранжевых теорий поля, разрешена таким образом.

\section{3. ХИГГСОВЫ ПОЛЯ НА КАЛИБРОВОЧНО-ЕСТЕСТВЕННОМ РАССЛОЕНИИ}

Обозначим через k алгебру Ли обобщенных векторных полей Якоби. Пусть $\mathfrak{h}$ - алгебра Ли правоинвариантных вертикальных векторных полей на $W^{(r+4 s, k+4 s)} \mathbf{P}$. Теперь предположим, что существуют глобальные решения обобщенных калибровочно-естественных уравнений Якоби. Тогда алгебра Ли k характеризуется как подалгебра Ли алгебры Ли $\mathfrak{h}$, морфизм Якоби является самосопряженным, а $\mathfrak{k}$ имеет постоянный ранг. Расщепленная структура $\mathfrak{h}=\mathfrak{k} \oplus \operatorname{Im} \mathcal{J}$ является корректно определенной и также редуктивной, $[\mathfrak{k}, \operatorname{Im} \mathcal{J}]=\operatorname{Im} \mathcal{J}[18]$. В частности, для каждого $\mathbf{p} \in W^{(r, k)} \mathbf{P}$ с помощью обозначений $\mathcal{W} \equiv \mathfrak{h}_{\mathbf{p}}, \mathcal{K} \equiv \mathfrak{k}_{\mathbf{p}}$ и $\mathcal{V} \equiv \operatorname{Im} \mathcal{J}_{\mathbf{p}}$ мы имеем разложение редуктивной алгебры Ли $\mathcal{W}=\mathcal{K} \oplus \mathcal{V}$, причем $[\mathcal{K}, \mathcal{V}]=\mathcal{V}$. Заметим, что $\mathcal{W}$ является алгеброй Ли группы Ли $W_{n}^{(r, k)} \mathbf{G}$.

Поскольку $\mathcal{K}$ является редуктивной алгеброй Ли алгебры Ли $\mathcal{W}$, существует изоморфизм между $\mathcal{V} \equiv \operatorname{Im} \mathcal{J}_{\mathbf{p}}$ и $T \mathbf{X}$, так что $\mathcal{V}$ оказывается образом горизонтального подпространства. Таким образом, мы характеризуем главное расслоение $\mathbf{Q} \rightarrow \mathbf{X}$, причем $\operatorname{dim} \mathbf{Q}=\operatorname{dim} \mathcal{W}$ так, что $\mathbf{X}=\mathbf{Q} / \mathbf{K}$. Главное подрасслоение $\mathbf{Q} \subset \mathbf{W}^{(r, k)} \mathbf{P}$ такое, что $\mathcal{K}=T_{\mathbf{q}} \mathbf{Q} / \mathbf{K}$, где $\mathbf{K}-$ (редуцированная) группа Ли алгебры Ли $\mathcal{K}$, поэтому является редуцированным главным расслоением.

Далее мы опустим порядки калибровочно-естественного продолжения для упрощения обозначений. Группа Ли $\mathbf{K}$ алгебры Ли $\mathcal{K}$ является, в частности, замкнутой подгруппой в $W \mathbf{G}$ (k - векторное подрасслоение). Мы имеем составное расслоение

$$
W \mathbf{P} \rightarrow W \mathbf{P} / \mathbf{K} \rightarrow \mathbf{X}
$$


где $W \mathbf{P} \rightarrow W \mathbf{P} / \mathbf{K}$ - главное расслоение со структурной группой $\mathbf{K}$, а $W \mathbf{P} / \mathbf{K} \rightarrow \mathbf{X}-$ расслоение, связанное с $W \mathbf{P}$, с типичным слоем $W \mathbf{G} / \mathbf{K}$, на который структурная группа $W \mathbf{G}$ действует слева. Таким образом,

$$
W \mathbf{P} / \mathbf{K}=W \mathbf{P} \underset{W \mathbf{G}}{\times} W \mathbf{G} / \mathbf{K} \rightarrow \mathbf{X}
$$

является калибровочно-естественным расслоением, функториально ассоциированным с $W \mathbf{P} \times W \mathbf{G} / \mathbf{K} \rightarrow \mathbf{X}$ с помощью правого действия $W \mathbf{G}$. Левое действие со стороны $W \mathbf{G}$ на $W \mathbf{G} / \mathbf{K}$ согласуется с разложением редуктивной алгебры Ли $\mathcal{W}=$ $\mathcal{K} \oplus \mathcal{V}$, причем $[\mathcal{K}, \mathcal{V}] \subset \mathcal{V}$. На самом деле векторное пространство $\mathcal{V}=\operatorname{Im} \mathcal{J}_{\mathbf{p}}=\mathcal{W} / \mathcal{K}$ содержит в себе левое действие $\mathcal{W}$, задаваемое с помощью сопряженного представления (так как $[\mathcal{K}, \mathcal{V}] \subset \mathcal{V}$, мы также имеем $[\mathcal{W}, \mathcal{V}]=[\mathcal{K} \oplus \mathcal{V}, \mathcal{V}]=[\mathcal{K}, \mathcal{V}] \oplus[\mathcal{V}, \mathcal{V}] \subset \mathcal{V})$.

Назовем глобальное сечение $h: \mathbf{X} \rightarrow W \mathbf{P} / \mathbf{K}$ калибровочно-естественным полем Хиггса [19]. Заметим, что это векторное поле, лежащее в образе морфизма Якоби (напомним, что $\mathcal{W} / \mathcal{K}=\mathcal{V}=\operatorname{Im} \mathcal{J}_{\mathbf{p}}$ ), который, в свою очередь, является образом горизонтального подпространства $T \mathbf{X}$. Прообраз расслоения

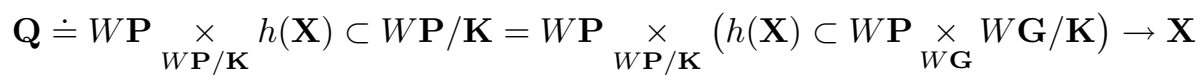

является редуцированным главным подрасслоением в Р. Заметим, что калибровочно-естественное поле Хиггса является глобальным сечением расслоения $\widehat{H}_{p}$, причем $\mathbf{p} \in \mathbf{Q}$.

Пусть $\omega$ - главная связность на $\mathbf{W}^{(r, k)} \mathbf{P}$, а $\bar{\omega}-$ главная связность на главном расслоении $\mathbf{Q}$, т. е. $\mathcal{K}$-инвариантное горизонтальное распределение, определяющее вертикальный параллелизм $\bar{\omega}: V \mathbf{Q} \rightarrow \mathcal{K}$ стандартным образом. Оно определяет расщепление $T_{\mathbf{p}} \mathbf{Q} \simeq_{\bar{\omega}} \mathcal{K} \oplus \widehat{H}_{\mathbf{p}}, \mathbf{p} \in \mathbf{Q}$. Так как $\mathcal{K}$ является подалгеброй алгебры Ли $\mathcal{W}$ и $\operatorname{dim} \mathbf{Q}=\operatorname{dim} \mathcal{W}$, определена главная картанова связность типа $\mathcal{W} / \mathcal{K}$, т. е. $\mathcal{W}$-значный абсолютный параллелизм $\widehat{\omega}: T \mathbf{Q} \rightarrow \mathcal{W}$, который является гомоморфизмом алгебр Ли при ограничении величиной $\mathcal{K}$. Скобки Ли сохраняются, если один из аргументов находится в $\mathcal{K}$ и $\widehat{\omega}$ является продолжением естественного вертикального параллелизма, т. е. $\left.\widehat{\omega}\right|_{V \mathbf{Q}}=\bar{\omega}$. В работе [7] мы определили $\widehat{\omega}$ как ограниченный величиной TQ естественный вертикальный параллелизм, определяемый главной связностью $\omega$ на $W^{(r, k)} \mathbf{P}$ с помощью фундаментального векторного отображения $\omega: V W^{(r, k)} \mathbf{P} \rightarrow \mathcal{W}$. Он удовлетворяет соотношению $H_{\mathbf{p}} \cap T_{\mathbf{p}} \mathbf{Q}=\varnothing$, при этом $\mathbf{p} \in \mathbf{Q}$, и является связностью на $\mathbf{W}^{(r, k)} \mathbf{P}=\mathbf{Q} \times_{\mathbf{K}} W_{n}^{(r, k)} \mathbf{G} \rightarrow \mathbf{X}$, т. е. картановой связностью на $\mathbf{Q} \rightarrow \mathbf{X}$ с значениями в $\mathcal{W}$. Заметим, что она расщепляется на $\mathcal{K}$-компоненту, которая является формой главной связности на $\mathcal{K}$-многообразии $\mathbf{Q}$, и на $\mathcal{V}$-компоненту, которая является формой смещения. Действительно, так как $\mathbf{K}$ является редуктивной подгруппой Ли в $\mathbf{W}_{n}^{(r, k)} \mathbf{G}$, главную картанову связность можно рассматривать как $\mathbf{K}$-структуру, снабженную главной формой связности $\eta=p r_{\mathcal{K}} \circ \widehat{\omega}$ на $\mathbf{Q}[20]$.

Калибровочно-естественное поле Хиггса, будучи глобальным сечением расслоения $\widehat{H}_{p}, \mathbf{p} \in \mathbf{Q}$, связано с формой смещения, определяемой с помощью $\mathcal{V}$-компоненты картановой связности $\widehat{\omega}$. Главное расслоение $W \mathbf{P}(\mathcal{W}$ - алгебра Ли структурной группы) допускает главное подрасслоение $\mathbf{Q}$ (с алгеброй Ли $\mathcal{K}$ структурной группы); более того, задана прямая сумма $\mathcal{W}=\mathcal{K} \oplus \mathcal{V}$, где $\mathcal{V}$ является таким подпространством, что $\operatorname{ad}(g)(\mathcal{V}) \subset \mathcal{V}, g \in \mathbf{K}$. Тогда полученный с помощью $h$ обратный образ 
$\mathcal{K}$-значной компоненты $\mathcal{W}$-значной главной связности $\omega$ на $W \mathbf{P}$ на редуцированном подрасслоении $\mathbf{Q}$ является формой связности главной связности на $\mathbf{Q}$ [21].

Если задано составное расслоение

$$
W \mathbf{P} \rightarrow W \mathbf{P} / \mathbf{K} \rightarrow \mathbf{X}
$$

то мы имеем точную последовательность

$$
0 \rightarrow V_{W \mathbf{P} / \mathbf{K}} W \mathbf{P} \rightarrow V W \mathbf{P} \rightarrow W \mathbf{P} \underset{W \mathbf{P} / \mathbf{K}}{\times} V W \mathbf{P} / \mathbf{K} \rightarrow 0
$$

где $V_{W \mathbf{P} / \mathbf{K}} W \mathbf{P}$ - вертикальное касательное расслоение к расслоению $W \mathbf{P} \rightarrow W \mathbf{P} / \mathbf{K}$. Каждая связность на последнем расслоении определяет расщепление

$$
V W \mathbf{P}=V_{W \mathbf{P} / \mathbf{K}} W \mathbf{P} \underset{W \mathbf{P} / \mathbf{K}}{\bigoplus} \widehat{\omega}(W \mathbf{P} \underset{W \mathbf{P} / \mathbf{K}}{\times} V W \mathbf{P} / \mathbf{K})
$$

с помощью которого мы можем определить вертикальный ковариантный дифференциал $\mathcal{D}: J^{1} W \mathbf{P} \rightarrow T^{*} \mathbf{X} \otimes_{W \mathbf{P}} V_{W \mathbf{P} / \mathbf{K}} W \mathbf{P}$, который связан с ковариантным дифференциалом на $W \mathbf{P}_{h}$ относительно связности обратного образа $h^{*}(\widetilde{\omega})$.

Поля Хиггса и производная Ли классических полей. В качестве примера рассмотрим случай производной Ли спинорных полей (этот пример изучался с новой точки зрения в работах [8], [17], а затем в работе [6], в контексте канонических связностей).

На четырехмерном многообразии, допускающем лоренцевы структуры $\left(S O(1,3)^{e}\right.$ редукции) $\mathbf{X}$, рассмотрим $S P I N(1,3)^{e}$-главное расслоение $\pi: \Sigma \rightarrow \mathbf{X}$ и отображение расслоений, индуцирующее спин-репер на $\Sigma$, задаваемый с помощью $\tilde{\Lambda}: \Sigma \rightarrow L(\mathbf{X})$, определяющие метрику $g$ с помощью редуцированного подрасслоения $S O(\mathbf{X}, g)=$ $\tilde{\Lambda}(\Sigma)$ расслоения $L(\mathbf{X})$. Левое действие $\rho$ группы $W^{(0,1)} \operatorname{SPIN}(1,3)^{e}$ на многообразии $G L(4, \mathbb{R})$ задается так, что ассоциированное расслоение $\Sigma_{\rho} \doteq W^{(0,1)} \Sigma \times_{\rho}$ $G L(4, \mathbb{R})$ является калибровочно-естественным расслоением порядка $(0,1)$ - расслоением спин-тетрад $\theta$ [22]. Индуцированная метрика записывается как $g_{\mu \nu}=\theta_{\mu}^{a} \theta_{\nu}^{b} \eta_{a b}$, где $\theta_{\mu}^{a}$ - локальные компоненты спин-тетрады $\theta$, а $\eta_{a b}$ - метрика Минковского. Пусть $\mathfrak{s o}(1,3) \simeq \mathfrak{s p i n}(1,3)$ - алгебра Ли группы $S O(1,3)$. Можно рассмотреть левое действие $W^{(1,1)} \operatorname{SPIN}(1,3)^{e}$ на векторном пространстве $\left(\mathbb{R}^{4}\right)^{*} \otimes \mathfrak{s o}(1,3)$. Ассоциированное расслоение $\Sigma_{l} \doteq W^{(1,1)} \Sigma \times_{l}\left(\left(\mathbb{R}^{4}\right)^{*} \otimes \mathfrak{s o}(1,3)\right)$ является калибровочно-естественным расслоением порядка $(1,1)$ - расслоением спиновых связностей $\omega$. Если $\widehat{\gamma}$ является линейным представлением $\operatorname{SPIN}(1,3)^{e}$ на векторном пространстве $\mathbb{C}^{4}$, индуцированным выбором матриц $\gamma$, то мы получаем калибровочно-естественное расслоение порядка $(0,0) \Sigma_{\widehat{\gamma}} \doteq \Sigma \times \widehat{\gamma} \mathbb{C}^{4}-$ расслоение спиноров. Спинорная связность $\widetilde{\omega}$ определяется стандартным образом через спиновую связность.

Далее, лагранжиан Эйнштейна-Картана является морфизмом, сохраняющим базис $\lambda_{\mathrm{EC}}: \Sigma_{\rho} \times_{\mathbf{X}} J_{1} \Sigma_{l} \rightarrow \Lambda^{4} T^{*} \mathbf{X}$, в то время как лагранжиан Дирака является морфизмом, сохраняющим базис $\lambda_{\mathrm{D}}: \Sigma_{\rho} \times \mathbf{X} \Sigma_{l} \times \mathbf{X} J_{1} \Sigma_{\widehat{\gamma}} \rightarrow \Lambda^{4} T^{*} \mathbf{X}$ (локальные выражения можно найти, например, в работе [5]). Мы полагаем, что полный лагранжиан гравитационного поля, взаимодействующего со спинорной материей, описывается выражением $\lambda=\lambda_{\mathrm{EC}}+\lambda_{\mathrm{D}}$. 
Пусть теперь $\bar{\Xi}-\operatorname{SPIN}(1,3)^{e}$-инвариантное векторное поле на $\Sigma$. Лагранжиан $\lambda$ инвариантен по отношению к любому подъему $\widehat{\Xi}$ поля $\bar{\Xi}$ на полное пространство теории. Согласно первой теореме Нётер можно найти сохраняющийся нётеровский ток $\epsilon(\lambda, \bar{\Xi})$ такой, что соответствующим суперпотенциалом будет $\nu(\lambda, \bar{\Xi}) \doteq-\frac{1}{2 k} \bar{\Xi}_{v}^{a b} \epsilon_{a b}$, где $\bar{\Xi}_{v b}^{a}=\bar{\Xi}_{b}^{a}-\omega_{b \mu}^{a} \xi^{\mu}-$ вертикальная часть поля $\bar{\Xi}$ по отношению к спиновой связности $\omega$.

Мы упоминали, что без априорной фиксации связности существование канонических глобальных сохраняющихся величин в теории поля связано с тождествами Нётер: в работе [8] мы обнаружили, что это означает, что $\bar{\Xi}_{v}^{a b}=-\tilde{\nabla}^{[a} \xi^{b]}$ (так называемый подъем Косманн [23]), где $\widetilde{\nabla}$ - ковариантная производная по отношению к стандартной транспонированной связности на $\Sigma_{\rho}$. Подъем Косманн может быть охарактеризован с вариационной точки зрения: он является единственным калибровочно-естественным подъемом, который гарантирует, что условие естественности (тождества Нётер) $\mathcal{L}_{j_{s+1}} \widehat{\Xi}_{\mathrm{H}}\left[\mathcal{L}_{j_{s+1}} \widehat{\Xi}_{\mathrm{V}} \lambda\right] \equiv 0$ выполняется. Вдоль такого подъема инвариантным является не только исходный лагранжиан $\lambda$, но также и его первая вариационная производная $\vartheta(\lambda, \mathfrak{K})$. С другой стороны, производная Ли спинорных полей может быть записана с помощью канонической спинорной связности $\widehat{\omega}$ следующим образом:

$$
£_{\bar{\Xi}} \psi=\xi^{\mu} \partial_{\mu} \psi+\frac{1}{4} \widehat{\Xi}_{h a b} \gamma^{a} \gamma^{b} \psi-\frac{1}{4} \nabla_{[a} \xi_{b]} \gamma^{a} \gamma^{b} \psi
$$

где $\widehat{\Xi}_{h}$ - горизонтальная часть поля $\widehat{\Xi}$ по отношению $к$ спинорной связности [6].

Ясно, что мы здесь рассматриваем редукцию полного главного расслоения, которое является основным структурным расслоением теории. Каждое глобальное сечение $h$ расслоения $W \mathbf{P} / \mathbf{K} \rightarrow \mathbf{X}$ (напомним, что $\mathbf{K}$ следует из калибровочно-естественных уравнений Якоби) позволяет определить вертикальный ковариантный дифференциал, который связан с вертикальным дифференциалом, определяемым главной связностью на полном калибровочно-естественном продолжении $W \mathbf{P}$, т. е. также и со спиновой и спинорной связностями, функториально индуцированными на ассоциированном расслоении, как было только что показано. В результате мы получили, что производная Ли полей является ограниченной и параметризуется полем Хиггса $h$, определяемым с помощью $\mathfrak{K}$. В частности, подъем Космана до полного расслоения тетрад и спиноров связан с вариационным полем Хиггса на калибровочно-естественном расслоении.

Благодарности. Исследование было выполнено при финансовой поддержке Туринского университета и MIUR и при частичной финансовой поддержке (М. Палезе) университета Брно (Чехия).

\section{Список литературы}

[1] D. J. Eck, Mem. Amer. Math. Soc., 33:247 (1981), 1-48.

[2] C. Ehresmann, C. R. Acad. Sci. Paris, 240 (1955), 1755-1757.

[3] I. Kolár, P. W. Michor, J. Slovák, Natural Operations in Differential Geometry, Springer, New York, 1993.

[4] M. Palese, E. Winterroth, Rep. Math. Phys., 54:3 (2004), 349-364, arXiv: math-ph/0406009. 
[5] L. Fatibene, M. Francaviglia, Natural and Gauge Natural Formalism for Classical Field Theories. A Geometric Perspective Including Spinors and Gauge Theories, Kluwer, Dordrecht, 2003.

[6] M. Ferraris, M. Francaviglia, M. Palese, E. Winterroth, Int. J. Geom. Methods Mod. Phys., 5:6 (2008), 973-988.

[7] M. Ferraris, M. Francaviglia, M. Palese, E. Winterroth, Int. J. Geom. Methods Mod. Phys., 8:1 (2011), 177-185.

[8] E.H.K. Winterroth, Variational derivatives of gauge-natural invariant Lagrangians and conservation laws, $\mathrm{PhD}$ thesis, University of Torino, 2007.

[9] D. Krupka, "Variational sequences on finite order jet spaces", Differential Geometry and Its Applications (Brno, Czechoslovakia, 27 Aug. - 2 Sept. 1989), eds. J. Janyška et al., World Sci., Singapore, 1990, 236-254.

[10] M. Palese, E. Winterroth, Arch. Math., 41:3 (2005), 289-310.

[11] М. Палезе, Е. Уинтеррот, ТМФ, 152:2 (2007), 377-389.

[12] P. G. Bergmann, Phys. Rev., 75:4 (1949), 680-685.

[13] E. Noether, Nachr. Akad. Wiss. Göttingen Math.-Phys. Kl. II, 2 (1918), 235-257.

[14] R. Utiyama, Phys. Rev., 101:5 (1956), 1597-1607.

[15] D. J. Saunders, The Geometry of Jet Bundles, London Math. Soc. Lecture Note Ser., Cambridge Univ. Press, Cambridge, 1989.

[16] R. Vitolo, Math. Proc. Cambridge Philos. Soc., 125:2 (1999), 321-333.

[17] M. Palese, E. Winterroth, "Noether identities in Einstein-Dirac theory and the Lie derivative of spinor fields", Proc. of the 10th International Conference on Differential Geometry and its Applications, DGA 2007 (Olomouc, Czech Republic, August 27-31, 2007), World Sci. Publ., Hackensack, NJ, 2008, 643-653.

[18] M. Palese, E. Winterroth, Rep. Math. Phys., 62:2 (2008), 229-239, arXiv: 0712.0925.

[19] G. Sardanashvily, Int. J. Geom. Methods Mod. Phys., 3:1 (2006), 139-148.

[20] L. Mangiarotti, G. Sardanashvily, Connections in Classical and Quantum Field Theory, World Sci. Publ., Singapore, 2000.

[21] M. Palese, E. Winterroth, "Invariant variational problems and Cartan connections on gauge-natural bundles", AIP Conf. Proc., 1191, eds. P. Kielanowski, Springer, Berlin, 2009, $160-165$.

[22] S. Weinberg, Gravitation and Cosmology: Principles and Applications of the General Theory of Relativity, Wiley, New York, 1972.

[23] Y. Kosmann, C. R. Acad. Sci. Paris Sér. A-B, 262 (1966), A289-A292; A394-A397. 